\title{
Protective effects of naringin in indomethacin-induced gastric ulcer in rats
}

\author{
Sengul Emin ${ }^{1,}{ }^{*}$ and Gelen Volkan ${ }^{2}$ \\ ${ }^{1}$ Atatürk University Veterinary Faculty, Department of Physiology, Erzurum, Turkey. \\ ${ }^{2}$ Kafkas University Veterinary Faculty, Department of Physiology, Kars, Turkey.
}

Publication history: Received on 12 July 2019; revised on 01 August 2019; accepted on 03 August 2019

Article DOI: https://doi.org/10.30574/gscbps.2019.8.2.0132

\begin{abstract}
This study aimed to investigate the potential protective effects of Naringin (NA) in Indomethacin (IND)-induced gastric ulcers in rats. Forty adult male Sprague Dawley rats were randomly divided into four groups; the control group was given intragastric (ig) saline for 10 days, the IND group was given ig saline for 10 days and given a single dose of IND $(100 \mathrm{mg} / \mathrm{kg}$, ig) on the 11th day, and the NA+IND and RAN+IND groups were administered NA (100 mg/kg, ig) and RAN (5mg/kg, ig) for 10 days, respectively. These final two groups were administered single dose IND (100 $\mathrm{mg} / \mathrm{kg}$, ig) on the 11th day, and 24 hours after IND administration, the rats were decapitated under anesthesia. The stomach tissues were examined to calculate the ulcer index and analyze inflammation and apoptosis markers. In addition, microscopic examination of the stomach tissues was performed. The results showed that IND induced gastric ulcers and increased tumor necrosis factor-alpha (TNF- $\alpha$ ), interleukin-6 (IL-6), C-reactive protein (CRP), inducible nitric oxide synthase (iNOS), and caspase-3 levels while decreasing cyclooxygenase-2 (COX-2). NA exhibited a protective effect by preventing these parameter changes. In addition, NA corrected the histopathological abnormalities caused by IND in stomach tissue. These results indicate that NA might protect against the effects of IND in rats.
\end{abstract}

Keywords: Gastric ulcer; Indomethacin; Naringin; Rat

\section{Introduction}

Nonsteroidal anti-inflammatory drugs (NSAIDs) such as indomethacin (IND) are extensively used to decrease pain and reduce fever in inflammatory diseases as rheumatoid arthritis and osteoarthritis. These drugs have side effects such as gastrointestinal toxicity and can cause peptic ulcers, bleeding and perforations in the digestive tract [1,2]. Nowadays, gastric ulcers are among the most common diseases; gastric acid, pepsin, bile salts, Helicobacter pylori infection, alcohol consumption and NSAIDs are major causes of gastric ulcers [3]. NSAIDs trigger gastric ulcers by causing damage to gastric mucosa [4]. At the same time, these drugs impair the balance between the healing of the damaged mucosa and endogenous angiogenesis [3]. Most patients must take additional drugs to prevent the side effects of NSAIDs [5]. IND is the NSAI compound with the highest ulcer potential [6]. The inhibition of cyclooxygenase $(\mathrm{COX})$, reduction of prostaglandin (PG) synthesis, oxidative stress and inflammation caused by IND are among the major causes of gastric pathogenesis $[7,8]$.

The protective or therapeutic effects of plant-based agents in experimental gastric ulcer models have been widely investigated in recent years [2, 5, 9]. Naringin (NA) is one of the flavonoid compounds used for this purpose [10], and NA is usually found in grapefruit, orange, and cooked tomato paste [11]. Flavonoids are secondary metabolites and sources of bioactive compounds in plants [12]. NA has anti-allergic, anti-ulcer, anti-inflammatory, anti-oxidant, antiapoptotic, anti-osteoporotic, and anti-cancer effects [13-16]. In the present study, the possible gastroprotective effects of NA were investigated in an experimental model of IND-induced gastric ulcer in rats.

\footnotetext{
${ }^{*}$ Corresponding author

E-mail address: emin.sengul@ataıni.edu.tr
} 


\section{Material and methods}

\subsection{Drugs}

IND, NA and Ranitidine (RAN) were purchased from Sigma Chemical Co. (St. Louis, Mo, U.S.A.).

\subsection{Animals}

In the study, was used 40 adult male Sprague Dawley rats, whose weights were 220-250 g. The care of the rats was done by providing proper moisture, light and room temperature, free water and feed intake until the day of the experiment. This study has been approved by the Animal Experiment Local Ethics Committee of Atatürk University (Protocol No: 2018/231).

\subsection{Experimental protocol}

The animals were randomly divided into four groups, consisting of a control group, IND group, NA+IND group and RAN+IND group. Control group was administered only intragastric (i.g.) saline for 10 days. IND group was given i.g. saline for 10 days for placebo and on eleventh days of study, single dose IND (100 mg/kg, i.g.) was administered. NA+IND group was administered NA $(100 \mathrm{mg} / \mathrm{kg}$, ig) for 10 days and RAN+IND group was given RAN (5mg/kg, ig) for 10 days. These two groups were given single dose IND $(100 \mathrm{mg} / \mathrm{kg}$, ig) on eleventh days. After 24 hours from IND administration, rats decapitated under anesthesia and taken stomachs. The ulcer index (UI) calculated to determine the severity of the IND-induced ulcer.

\subsection{Analysis of cytokines levels and iNOS activity}

Stomach tissues were homogenized in phosphate buffer saline (pH: 7.2-7.4) using homogenizer. Afterwards, they were centrifuged at $5000 \mathrm{rpm}$ at $4{ }^{\circ} \mathrm{C}$ for $10 \mathrm{~min}$ for collection of the supernatants. The supernatants were used to detect the expression of tumor necrosis factor-alpha (TNF- $\alpha$ ), interleukin-6 (IL-6), C-reactive protein (CRP), inducible nitric oxide synthase (iNOS) and using enzyme-linked immunosorbent assay (ELISA) kits according to the manufacturer's instructions (Cayman and Elabscience, Wuhan, China).

\subsection{Analysis of gastric apoptosis}

Caspase-3 activity was determined by means of ELISA immunoassay kits. According to the manufacturer's protocol, the results were expressed as mean $\pm \mathrm{SD}$ of the activity of each factor in the tissues.

\subsection{Histopathological examination}

Stomach tissues were fixed in $10 \%$ formalin for 48 hours and embedded in paraffin after routine processing. The sections $(4 \mu \mathrm{m})$ were cut with microtome, stained with hematoxylin-eosin (HE) and assessed under an Olympus microscope (Leica DM 1000, Germany). The sections were evaluated as non-positive (-), mild (+), moderate (++) and severe $(+++)$.

\subsection{Statistical analysis}

All data were statistically evaluated in one-way ANOVA by using SPSS 20.00. The post-hoc Tukey test was used to compare the values between the groups. The data were expressed as mean \pm standard deviation (SD). $\mathrm{p}<0.05$ was considered statistically significant.

\section{Results}

\subsection{Effects of NA on the severity of gastric lesion}

Treatment of rats with IND caused a significant increase in the UI in comparison to controls (Table 1, Figure 1 and 2 , ***: $\mathrm{P}<0.001, \mathrm{n}=10$ ). The pretreatment with both NA and RAN significantly decreased (by $73.66 \%$ and $75.71 \%$, respectively) the UI caused by IND and UI in these groups higher than control (Table 1, Figure 1 and $2, * *$ : P<0.05, $\mathrm{n}=10$ ). 
Table 1 Ulcer index and preventive index in the studied groups

\begin{tabular}{lll}
\hline Groups & Ulcer Index & Preventive Index \\
\hline Control & - & - \\
IND & $12.19 \pm 1.67^{\mathrm{a}}$ & - \\
NA+IND & $3.21 \pm 0.62^{\mathrm{b}}$ & $73.66 \%$ \\
RAN+IND & $2.96 \pm 0.46^{\mathrm{b}}$ & $75.71 \%$ \\
\hline \multicolumn{2}{c}{$\mathrm{b}: \mathrm{p}<0.001$}
\end{tabular}
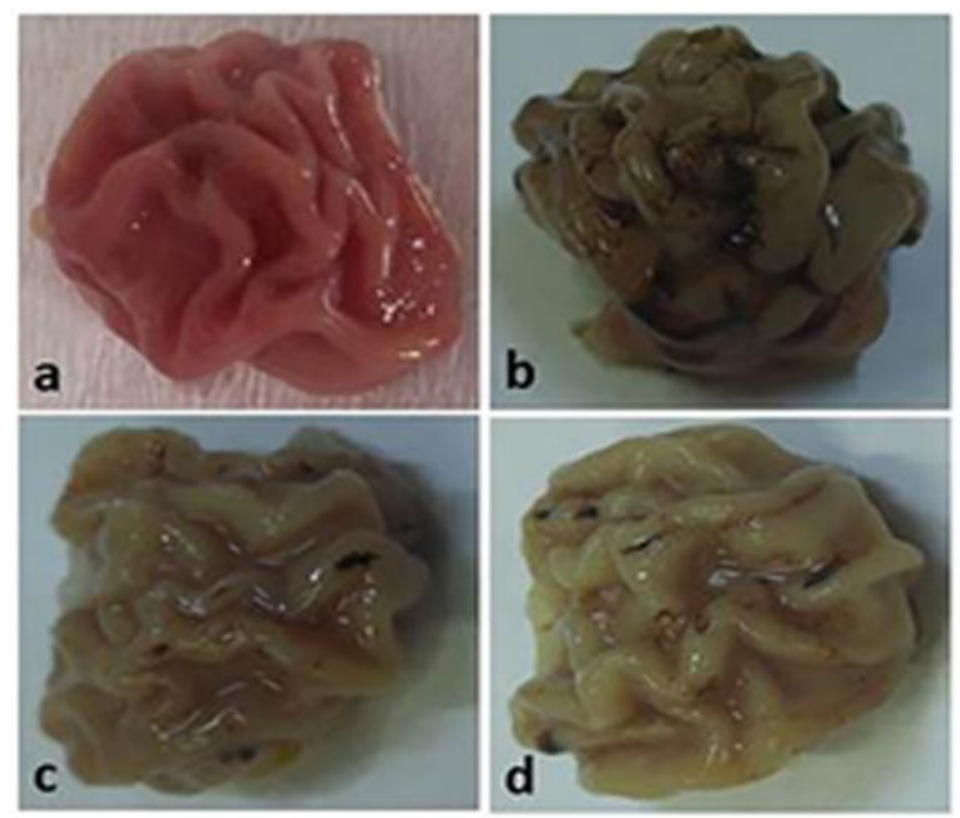

Figure 1 Effect of NA and RAN on the severity of gastric lesion (gross examination) examined in IND-induced gastric ulceration model. Control (a), IND (b), NA+IND (c), RAN+IND (d).

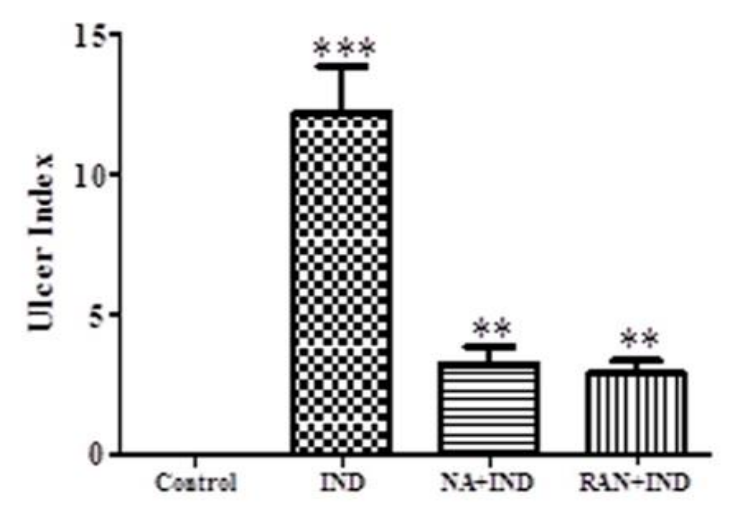

Figure 2 Effect of NA on the severity of gastric lesion (UI) in IND-induced gastric ulcer

IND caused significant decreases in gastric COX-2 levels in comparison to the controls. NA and RAN application prevented a decline in COX-2 levels, but COX-2 levels in these two groups were lower than those of the control group (Figure $3{ }^{* * *}: \mathrm{p}<0.0001,{ }^{* *}: \mathrm{p}<0.001, \mathrm{n}=10$ ). 


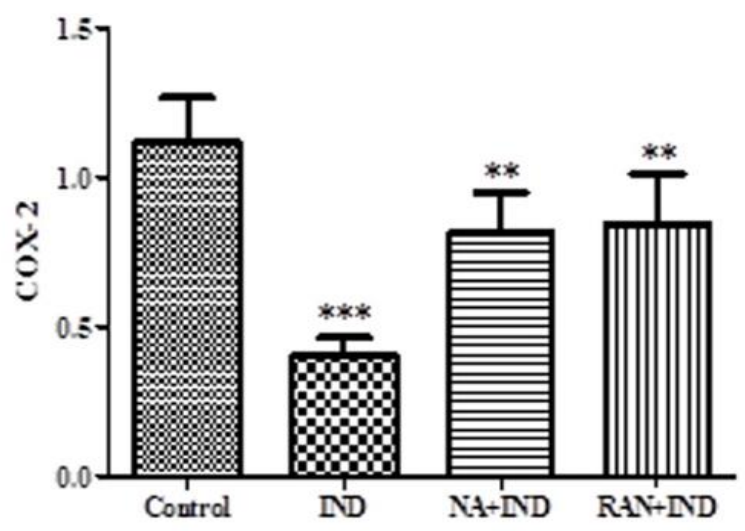

Figure 3 COX-2 levels in experimental groups (***: $\mathrm{p}<0.0001,{ }^{* *}: \mathrm{p}<0.001, \mathrm{n}=10$ )

IND administration significantly increased TNF- $\alpha$ and IL-6 levels compared to the controls, and NA and RAN treatment significantly prevented this increase. In addition, the TNF- $\alpha$ levels in the NA and RAN groups were higher than those of controls. RAN administration exhibited more effective prevention of the increase in IL-6 levels than NA (Figure 4A and $\left.4 \mathrm{~B},{ }^{* * *}: \mathrm{p}<0.0001, *: \mathrm{p}<0.05, \mathrm{n}=10\right)$. CRP and iNOS levels in the IND group were significantly higher than those in the other groups (Figure 4C and 4D, ${ }^{* * *}: \mathrm{p}<0.0001, \mathrm{n}=10$ ).
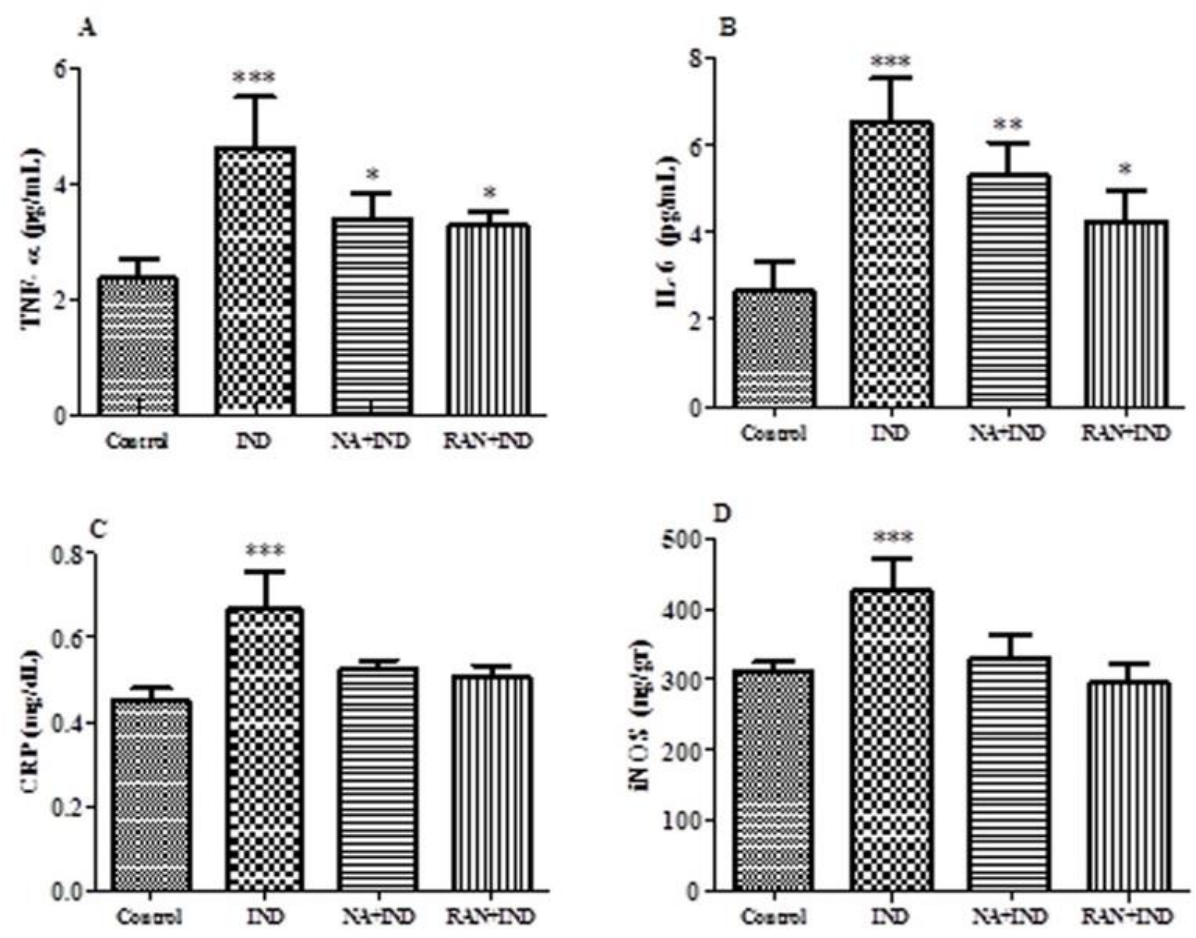

Figure 4 Effects of NA on TNF- $\alpha$ (A), IL-6 (B), CRP (C) and iNOS (D) parameters in IND-induced gastric ulcer in rats $(* * *: p<0.0001, * *: p<0.001, *: p<0.05, n=10)$

The caspase-3 expression in the IND group was significantly higher than that in controls. NA and RAN treatment markedly prevented the increase of caspase-3 expression in stomach tissue, and it was determined that caspase- 3 expression in these groups did not differ significantly than that in controls (Figure 5, ***: $\mathrm{p}<0.0001, \mathrm{n}=10$ ). 


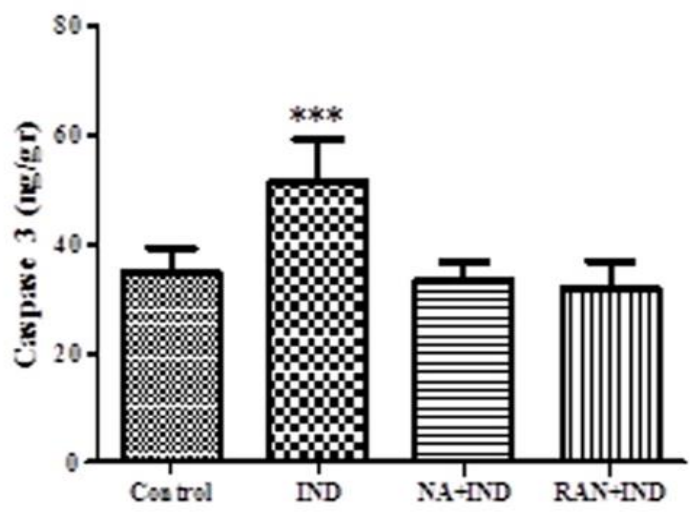

Figure 5 Caspase 3 expression in stomach tissues of experimental groups $(* * *: p<0.0001, \mathrm{n}=10)$

\subsection{Histopathology}

When the gastric tissues of the rats in the control group were examined, all the layers were observed to be normal in histological appearance (Figure 6A). In the IND group, severe hemorrhage was identified in the mucosa, as well as erosion in the mucosal epithelium, desquamation, deep necrosis focus, and mononuclear cell infiltrations. In addition, severe edema and hyperemia were detected in the submucosa and the vessels, respectively (Figure 6B). In the gastric mucosal epithelia of the rats treated with NA, slight erosion and necrosis were detected, mild hyperemia was detected in the vessels, and slight edema was detected in the submucosa (Figure 6C). RAN-treated rats showed slight erosion and epithelial necrosis in the gastric mucosa. Moderate hyperemia, edema, and mild mononuclear cell infiltration were also detected in the submucosa (Figure 6D). Histopathological findings were summarized in Table 2.

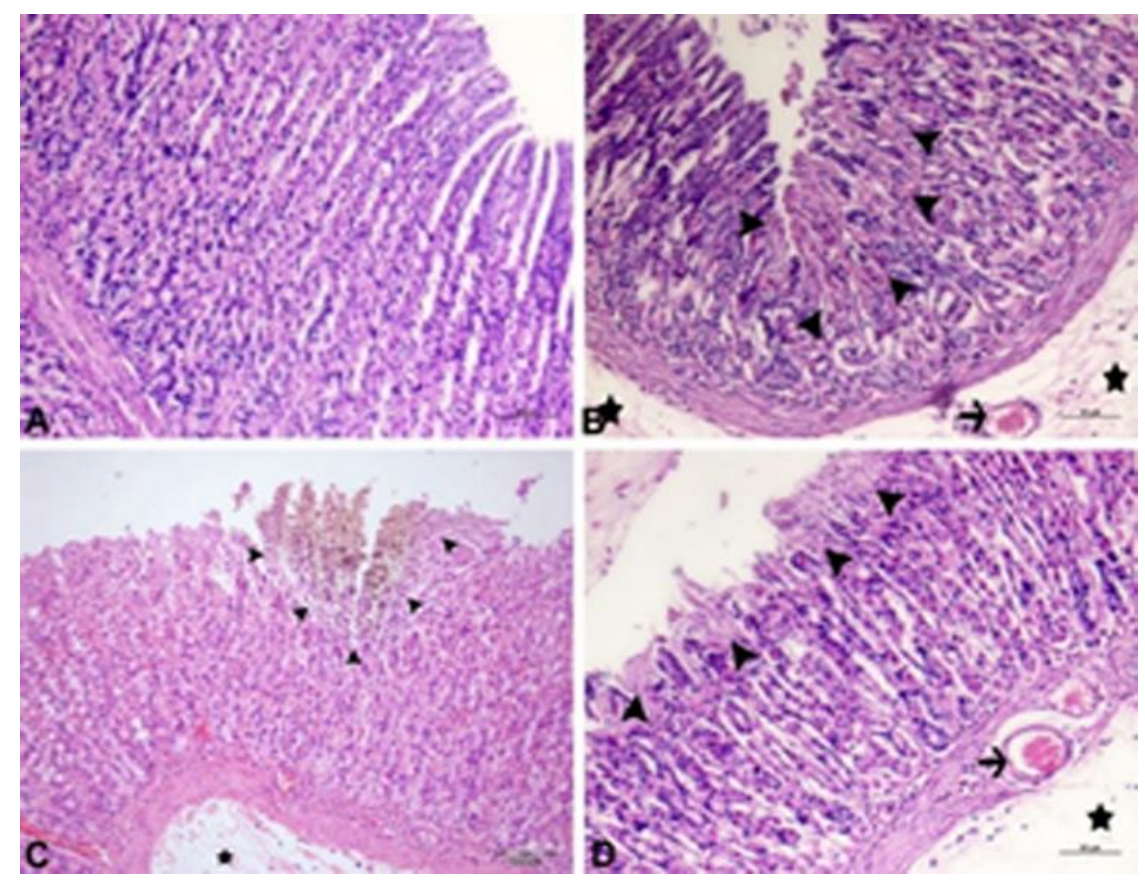

Figure 6 Control (A), normal histological appearance in stomach tissue; IND (B), deep erosion and necrosis in gastric mucosa (arrowheads), severe hyperemia in the vessels in the mucosa and submucosa (arrow), mononuclear cell infiltration, severe edema in the submucosa (stars); NA+IND (C), very mild erosion in gastric mucosa and necrosis (arrowheads), hyperemia the vessels and mild edema in the submucosa (asterisk); RAN+IND (D), mild erosion and necrosis in gastric mucosa, hyperemia in the vessels (arrow) and mild edema in the submucosa (asterisk), H\&E, Bar: $20 \mu \mathrm{m}$. 
Table 2 Histopathological findings in experimental groups

\begin{tabular}{|c|c|c|c|c|c|}
\hline & & Control & IND & NA+IND & RAN+IND \\
\hline $\begin{array}{l}\text { Mucosal } \\
\text { Erosion }\end{array}$ & Epithelium & - & +++ & ++ & ++ \\
\hline $\begin{array}{l}\text { Mucosal } \\
\text { Necrosis }\end{array}$ & Epithelium & - & +++ & + & + \\
\hline \multicolumn{2}{|c|}{ Vessel Hyperemia } & - & +++ & ++ & +++ \\
\hline \multicolumn{2}{|c|}{ Mucosal Edema } & - & +++ & ++ & ++ \\
\hline
\end{tabular}

-: non-positive, +: mild, ++: moderate and +++: severe

\section{Discussion}

NSAIDs have been extensively used clinically as anti-inflammatory and analgesic agents. However, ulcerative lesions of the gastrointestinal tract are a major side effect of these drugs and are the major limitation to their use $[17,18]$. This study investigated the gastroprotective effects of NA in an IND-induced gastric acid model in rats. We determined that NA prevented ulcer formation, inflammation and apoptosis, and for these reasons, it significantly inhibited INDinduced ulcers in rats.

IND, a noncorticosteroid drug, induces ulcerative gastric damage. Polar lipids in the structure of IND have an affinity for the lipophilic areas of cell membranes. These polar lipids cause disruption in the cell membrane and decrement of structural phospholipids and membrane proteins. Consequently, the reduced hydrophobicity of the gastric mucosal surface facilitates the penetration of some agents (gastric acid, pepsin, bile salts) into the mucosa. These agents cause lipid peroxidation in the mucosa and play an important role in the development of gastric mucosal lesions [17-19]. IND causes ulcerations with hemorrhages and significant increases in gastric UI in normal rats. Our results were in agreement with previous studies [17-20] and we determined that UI in the IND group was significantly higher than in the control and other groups. Administration of NA prevented gastric ulcers; thus, UI was lower than in the toxicity group.

IND causes gastric ulcers by inducing various events, such as the generation of reactive oxygen species (ROS), the triggering of lipid peroxidation, the induction of apoptosis and the inhibition of prostaglandin E2 [19]. NSAIDs induce gastric injury partly by inhibiting the cyclooxygenases (COX). COX-1 regulates basal prostanoid levels, while COX-2 is associated with the inflammatory response [21]. NSAIDs induce gastric injury partly by inhibiting the cyclooxygenases (COX). COX-1 regulates basal prostanoid levels, while COX-2 is associated with the inflammatory response [4]. When COX activity is blocked by IND, the PG level decreases and impairs gastroprotection mechanisms, reducing mucus and bicarbonate secretion, decreasing mucosal blood flow and leucocyte infiltration, deteriorating microvascular structures and increasing gastric acid secretions [22]. In our study, COX-2 levels were lower than control in INDtreated groups, and NA significantly prevented decreases in COX-2 levels; these results were in agreement with previous research $[5,23]$.

NSAIDs may increase the synthesis of TNF- $\alpha$ and leukotrienes [24,25], and some clinical studies have determined that the levels of inflammatory factors such as TNF- $\alpha$, IL-6, and CRP were compatible with the condition of gastric ulcer patients [26]. TNF- $\alpha$ and IL-6 are important mediators in NSAID-induced gastropathy; its production is normally inhibited by an increase in PGE2 levels [27]. CRP is a positive predictive parameter for gastric ulcers caused by active inflammation [28]. TNF- $\alpha$ may have the ability to activate pro-apoptotic caspases [29]. A previous study determined that IND treatment significantly elevated TNF- $\alpha$ levels in rats [24]. Moreover, pretreatment with dexamethasone and PGE2 could prevent increases in gastric injuries and TNF- $\alpha$ levels. Abbas et al. [9] determined that IND administration increased TNF- $\alpha$ levels while decreasing PGE2 levels. In other studies, increased TNF- $\alpha$ levels in IND-treated rats were observed [5]. Li et al. [30] detected that TNF- $\alpha$, IL-6, and CRP levels were increased in gastric ulcers. In accordance with the literature, in the present study, IND administration increased TNF- $\alpha$, IL-6, and CRP levels, and NA treatment prevented these increases.

In gastric ulcers induced by ethanol and IND, iNOS levels increase. Guo et al. [21] determined that iNOS expression was associated with severe inflammation in ulcer tissue. Lee et al. [31] and Pan et al. [32] reported that iNOS levels were increased in ethanol-induced gastric ulcers. The results of the present study have shown that iNOS levels 
increased in the IND group, and NA treatment prevented this increase. NA may decrease NO production via iNOS inhibition in tissue.

The balance between proliferation and death of gastric epithelial cell is of tremendously importance in sustaining of gastric mucosal integrity and apoptosis is regulated by caspase and Bcl-2 families [33]. Caspase-3 is a key mediator for apoptosis and Liu et al. [34] determined that caspase-3 is play in apoptotic cell death in stress-induced ulcer rat model. Some studies have shown that caspase 3 expression has increased in experimentally gastric ulcer models [3538]. We detected that IND induced to apoptosis by increasing caspase-3 expression in stomach tissue and NA administration prevented to this evaluate in caspase-3 expression.

IND causes gastric lesions, erosions, and ulcers; congestion; severe alterations in the glandular parts; hemorrhages; the depletion of the gastric mucus layer; amyloidosis; and infiltration of inflammatory cells [39, 40]. In the histopathological examination of the stomach tissues, we found ulcer injuries, severe hemorrhages in the mucosa, erosion in the mucosa epithelium, desquamation and mononuclear cell infiltrations. Our findings were in line with previous reports [40-42] and these observed changes were consistent with the significant elevation of the gastric level of TNF- $\alpha$ measured. TNF- $\alpha$ is known to play an important role in the pathophysiology of IND-induced gastric ulcers, and it causes the activation of neutrophil infiltration [43]. Also, we determined that NA and RAN treatment significantly prevented these pathological changes.

\section{Conclusion}

In the present study determined that IND administration induced to gastric ulcer in rats and NA has protective effects due to anti-inflammatory and anti-apoptotic effects.

\section{Compliance with ethical standards}

\section{Acknowledgments}

We thanks to Assoc. Prof. Serkan Yildirim due to his contributions in histopathology studies.

\section{Disclosure of conflict of interest}

Emin Sengul and Volkan Gelen declare that have no conflict of interest.

\section{Statement of ethical approval}

This study has been approved by the Animal Experiment Local Ethics Committee of Atatürk University (Protocol No: 2018/231).

\section{References}

[1] Teichert M, Griens F, Buijs E, Wensing M and De Smet P. (2014). Effectiveness of interventions by community pharmacists to reduce risk of gastrointestinal side effects in nonselective nonsteroidal anti-inflammatory drug users. Pharmacoepidemiology and Drug Safety, 23(4), 382-389.

[2] Morsy MA and El-Moselhy MA. (2013). Mechanisms of the protective effects of curcumin against indomethacininduced gastric ulcer in rats. Pharmacology, 91(5-6), 267-274.

[3] Musumba C, Pritchard DM and Pirmohamed M. (2009). Cellular and molecular mechanisms of NSAID-induced peptic ulcers. Alimentary Pharmacology and Therapeutics, 30(6), 517-531.

[4] Fornai M, Antonioli L, Colucci R, Tuccori M and Blandizzi C. (2011). Pathophysiology of gastric ulcer development and healing: molecular mechanisms and novel therapeutic options. InTech, 7, 113-142.

[5] Wu JZ, Liu YH, Liang JL, Huang QH, Dou YX, Nie J, et al. (2018). Protective role of $\beta$-patchoulene from Pogostemon cablin against indomethacin-induced gastric ulcer in rats: Involvement of anti-inflammation and angiogenesis. Phytomedicine, 39, 111-118.

[6] Al Asmari A, Al Omani S, Al Otaibi M, Al Abdulaaly AA, Elfaki I, Al Yahya K, et al. (2014). Gastroprotective effect of minocycline in experimentally induced gastric ulcers in rats. International Journal of Clinical and Experimental Medicine, 7(3), 586. 
[7] Yadav SK, Adhikary B, Chand S, Maity B, Bandyopadhyay SK and Chattopadhyay S. (2012). Molecular mechanism of indomethacin-induced gastropathy. Free Radical Biology \& Medicine, 12- 52(7), 1175-1187.

[8] Takeuchi K. (2012). Pathogenesis of NSAID-induced gastric damage: importance of cyclooxygenase inhibition and gastric hypermotility. World Journal of Gastroenterology, 18(18), 2147.

[9] Abbas AM and Sakr HF. (2013). Effect of selenium and grape seed extract on indomethacin-induced gastric ulcers in rats. Journal of Physiology and Biochemistry, 69(3), 527-537.

[10] Martin MJ, Marhuenda E, Perez-Guerrero C and Franco JM. (1994). Antiulcer effect of naringin on gastric lesions induced by ethanol in rats. Pharmacology, 49(3), 144-150.

[11] Igual M, Garcia-Martinez E, Camacho MM and Martinez Navarrete N. (2013). Jam processing and storage effects on $\beta$-carotene and flavonoids content in grapefruit. Journal of Functional Foods, 5, 736-744.

[12] Ghasemzadeh A and Jaafar HZ. (2013). Profiling of phenolic compounds and their antioxidant and anticancer activities in pandan (Pandanus amaryllifolius Roxb.) extracts from different locations of Malaysia. BMC Complementary and Alternative Medicine, 13, 341.

[13] Benavente-García 0 and Castillo J. (2008). Update on uses and properties of citrus flavonoids: new findings in anticancer, cardiovascular, and antiinflammatory activity. Journal of Agricultural and Food Chemistry, 56(15), 6185-6205.

[14] Mohamed EA, Hashim IIA, Yusif RM, Shaaban AAA, El-Sheakh AR, Hamed MF, et al. (2018). Polymeric micelles for potentiated antiulcer and anticancer activities of naringin. International Journal of Nanomedicine, 13, 1009.

[15] Gelen V, Șengül E, Yıldırım S and Atila G. (2018). The protective effects of naringin against 5-fluorouracilinduced hepatotoxicity and nephrotoxicity in rats. Iranian Journal of Basic Medical Sciences, 21(4), 404.

[16] Wang DM, Yang YJ, Zhang L, Zhang X, Guan FF and Zhang LF. (2013). Naringin enhances CaMKII activity and improves long-term memory in a mouse model of Alzheimer's disease. International Journal of Molecular Sciences, 14, 5576-5586.

[17] Kim JH, Kim BW, Kwon HJ and Nam SW. (2011). Curative effect of selenium against indomethacin-induced gastric ulcers in rats. Journal of Microbiology and Biotechnology, 21(4), 400-404.

[18] Suleyman B, Halici Z, Odabasoglu F and Gocer F. (2012). The effect of lacidipine on indomethacin induced ulcers in rats. International Journal of Pharmacology, 8(2), 115-121.

[19] Sabina EP and Rasool M. (2007). Therapeutic efficacy of Indian ayurvedic herbal formulation triphala on lipid peroxidation, antioxidant status and inflammatory mediator TNF-a in adjuvant-induced arthritic mice. International Journal of Radiation Biology, 1, 149-155.

[20] Abdallah IZA, Khattab HAH and Heeba GH. (2011). Gastroprotective effect of Cordia myxa L. fruit extract against indomethacin-induced gastric ulceration in rats. Life Science Journal, 8(3), 433-445.

[21] Takeuchi K. (2012). Pathogenesis of NSAID-induced gastric damage: Importance of cyclooxygenase inhibition and gastric hypermotility. World Journal of Gastroenterology, 18, 2147-2160.

[22] Jainu M, Mohan V and Devi S. (2006). Protective effect of Cissus quadrangularis on neutrophil mediated tissue injury induced by aspirin in rats. Journal of Ethnopharmacology, 104(3), 302-305.

[23] Chatterjee A, Chatterjee S, Das S, Saha A, Chattopadhyay S and Bandyopadhyay SK. (2012). Ellagic acid facilitates indomethacin-induced gastric ulcer healing via COX-2 up-regulation. Acta Biochimica et Biophysica Sinica, 44(7), 565-576.

[24] Appleyard CB, McCafferty, DM, Tigley AW, Swain MG and Wallace JL. (1996). Tumor necrosis factor mediation of NSAID-induced gastric damage: role of leukocyte adherence. American Journal of Physiology, 270, 42-48.

[25] Rainsford KD. (1983). Microvascular injury during gastric mucosal damage by anti-inflammatory drugs in pigs and rats. Inflammation Research, 13(5), 457-460.

[26] Zhang Y. (2013). Impact of esomeprazole on gastrointestinal hormones and inflammatory state of patients with gastric ulcer. Journal of Clinical Medicine Research, 17, 68-70.

[27] Kast RE. (2000). Tumor necrosis factor has positive and negative self-regulatory feedback cycles centered around cAMP. International Immunopharmacology, 22(11), 1001-1006. 
[28] Boehme MW, Autschbach F, Ell C and Raeth U. (2007). Prevalence of silent gastric ulcer, erosions or severe acute gastritis in patients with type 2 diabetes mellitus-a cross-sectional study. Hepatogastroenterology, 54, 643-648.

[29] Konturek PC, Duda A, Brzozowski T, Konturek SJ, Kwiecien S, Drozdowicz D, et al. (2000). Activation of genes for superoxide dismutase, interleukin-1beta, tumor necrosis factor-alpha, and intercellular adhesion molecule1 during healing of ischemia-reperfusion-induced gastric injury. Scandinavian Journal of Gastroenterology, 35, 452-463.

[30] Li XM, Miao Y, Su QY, Yao JC, Li HH and Zhang GM. (2016). Gastroprotective effects of arctigenin of Arctium lappa L. on a rat model of gastric ulcers. Biomedical Reports, 5(5), 589-594.

[31] Lee KP, Choi NH, Sudjarwo GW, Ahn SH, Park IS, Lee SR, et al. (2016). Protective effect of Areca catechu leaf ethanol extract against ethanol-induced gastric ulcers in ICR mice. Journal of Medicinal Food, 19(2), 127-132.

[32] Pan LR, Tang Q, Fu Q, Hu BR, Xiang JZ and Qian JQ. (2005). Roles of nitric oxide in protective effect of berberine in ethanol-induced gastric ulcer mice. Acta Pharmacologica Sinica, 26(11), 1334.

[33] Colucci R, Fornai M, Antonioli L, Ghisu N, Tuccori M, Blandizzi C, et al. (2009). Characterization of mechanisms underlying the effects of esomeprazole on the impairment of gastric ulcer healing with addition of NSAID treatment. Digestive and Liver Disease, 41(6), 395-405.

[34] Liu X, Chen Z, Mao N and Xie Y. (2012). The protective of hydrogen on stress-induced gastric ulceration. International Immunopharmacology, 13(2), 197-203.

[35] Luo XJ, Liu B, Dai Z, Li TB, Li NS, et al. (2013). Expression of apoptosis-associated microRNAs in ethanolinduced acute gastric mucosal injury via JNK pathway. Alcohol, 47, 481-493.

[36] Arab HH, Salama SA, Omar HA, Arafa el-SA and Maghrabi IA. (2015). Diosmin protects against ethanol-induced gastric injury in rats: novel anti-ulcer actions. PLoS One, 10(3), 0122417.

[37] Yu J, Peng H, Lin Y and Yi S. (2013). Effect of moxibustion treatment on cell apoptosis and expressions of heat shock protein and second mitochondrial activator of caspase in acute gastric mucosal lesion of rats. Journal of Traditional Chinese Medicine, 33, 258-261.

[38] Soliman NA, Zineldeen DH, Katary MA and Ali DA. (2016). N-acetylcysteine a possible protector against indomethacin-induced peptic ulcer: crosstalk between antioxidant, anti-inflammatory, and antiapoptotic mechanisms. Canadian Journal of Biochemistry and Physiology, 95(4), 396-403.

[39] Thong-Ngam D, Choochuai S, Patumraj S, Chayanupatkul M and Klaikeaw N. (2012). Curcumin prevents indomethacin-induced gastropathy in rats. World Journal of Gastroenterology, 18(13), 1479.

[40] Koc K, Cerig S, Ucar S, Colak S, Bakir M, Erol HS, et al. (2018). Gastroprotective effects of oleuropein and thymol on indomethacin-induced gastric ulcer in Sprague-Dawley rats. Drug and Chemical Toxicology, 1-13.

[41] Kanter M, Demir H, Karakaya C and Ozbek H. (2005). Gastroprotective activity of Nigella sativa L oil and its constituent, thymoquinone against acute alcohol-induced gastric mucosal injury in rats. World Journal of Gastroenterology, 11(42), 6662.

[42] Birdane FM, Cemek M, Birdane YO, Gülçin İ and Büyükokuroğlu ME. (2007). Beneficial effects of Foeniculum vulgare on ethanol-induced acute gastric mucosal injury in rats. World Journal of Gastroenterology, 13(4), 607.

[43] Suleyman H, Albayrak A, Bilici M, Cadirci E and Halici Z. (2010). Different mechanisms in formation and prevention of indomethacin-induced gastric ulcers. Inflammation, 33(4), 224-234.

\section{How to cite this article}

Sengul E and Gelen V. (2019). Protective effects of naringin in indomethacin-induced gastric ulcer in rats. GSC Biological and Pharmaceutical Sciences, 8(2), 06-14. 